\title{
Affine algorithms for the split variational inequality and equilibrium problems
}

Yonghong Yao ${ }^{1 *}$, Rudong Chen ${ }^{1}$ and Yeong-Cheng Liou ${ }^{2}$

\section{"Correspondence:}

yaoyonghong@aliyun.com

'Department of Mathematics,

Tianjin Polytechnic University,

Tianjin, 300387, China

Full list of author information is

available at the end of the article

\section{Abstract \\ An affine algorithm for the split variational inequality and equilibrium problems is presented. Strong convergence result is given.}

Keywords: affine algorithm; split method; variational inequality; equilibrium problem

\section{Introduction}

In the present manuscript, we focus on the following split variational inequality and equilibrium problem: Finding a point $x^{*}$ such that

$$
x^{*} \in \operatorname{GVI}(B, \psi, C) \text { and } \psi\left(x^{*}\right) \in \operatorname{EP}(F, A) \text {, }
$$

where $\operatorname{GVI}(B, \psi, C)$ is the solution set of the generalized variational inequality of finding $u \in C, \psi(u) \in C$ such that

$$
\langle B u, \psi(v)-\psi(u)| \geq 0, \quad \forall \psi(v) \in C,
$$

and $\operatorname{EP}(F, A)$ is the solution set of the equilibrium problem, which is to find $x^{\dagger} \in C$ such that

$$
F\left(x^{\dagger}, y\right)+\left\langle A x^{\dagger}, y-x^{\dagger}\right\rangle \geq 0, \quad \forall y \in C .
$$

Our main motivations are inspired by the following reasons.

Reason 1 Recently, the split problems have been considered by some authors. Especially, the split feasibility problem which can mathematically be formulated as the problem of finding a point $\tilde{x}$ with the property

$$
\tilde{x} \in C \quad \text { and } \quad g(\tilde{x}) \in Q
$$

has received much attention due to its applications in signal processing and image reconstruction with particular progress in intensity modulated radiation therapy [1-13]. Note that the involved operator $g$ is a bounded linear operator. However, in the present paper, the involved mapping $\psi$ in (1.1) is a nonlinear mapping.

(c) 2013 Yao et al.; licensee Springer. This is an Open Access article distributed under the terms of the Creative Commons Attribution License (http://creativecommons.org/licenses/by/2.0), which permits unrestricted use, distribution, and reproduction in any medium, provided the original work is properly cited. 
Reason 2 The variational inequality problem [14-24] and equilibrium problem [23-27], which include the fixed point problems and optimization problems [28-30], have been studied by many authors. It is an interesting topic associated with the analytical and algorithmic approach to the variational inequality and equilibrium problems.

Motivated and inspired by the results in the literature, we present an affine algorithm for solving the split problem (1.1). Strong convergence theorem is given under some mild assumptions.

\section{Preliminaries}

Let $H$ be a real Hilbert space with the inner product $\langle\cdot, \cdot \cdot\rangle$ and the norm $\|\cdot\|$, respectively. Let $C$ be a nonempty closed convex subset of $H$.

\subsection{Monotonicity and convexity}

An operator $A: C \rightarrow H$ is said to be monotone if $\langle x-y, A x-A y\rangle \geq 0$ for all $x, y \in C$. $A: C \rightarrow H$ is said to be strongly monotone if there exists a constant $\gamma>0$ such that $\langle x-$ $y, A x-A y\rangle \geq \gamma\|x-y\|^{2}$ for all $x, y \in C . A: C \rightarrow H$ is called an inverse-strongly-monotone operator if there exists $\alpha>0$ such that $\langle x-y, A x-A y\rangle \geq \alpha\|A x-A y\|^{2}$ for all $x, y \in C$. Let $g: C \rightarrow C$ be a nonlinear operator. $A: C \rightarrow H$ is said to be $\alpha$-inverse strongly $g$-monotone iff $\langle g(x)-g(y), A x-A y\rangle \geq \alpha\|A x-A y\|^{2}$ for all $x, y \in C$ and for some $\alpha>0$. Let $B$ be a mapping of $H$ into $2^{H}$. The effective domain of $B$ is denoted by $\operatorname{dom}(B)$, that is, $\operatorname{dom}(B)=$ $\{x \in H: B x \neq \emptyset\}$. A multi-valued mapping $B$ is said to be a monotone operator on $H$ iff $\langle x-y, u-v\rangle \geq 0$ for all $x, y \in \operatorname{dom}(B), u \in B x$, and $v \in B y$. A monotone operator $B$ on $H$ is said to be maximal iff its graph is not strictly contained in the graph of any other monotone operator on $H$.

A function $F: H \rightarrow R$ is said to be convex if for any $x, y \in H$ and for any $\lambda \in[0,1], F(\lambda x+$ $(1-\lambda) y) \leq \lambda F(x)+(1-\lambda) F(y)$.

\subsection{Nonexpansivity and continuity}

A mapping $T: C \rightarrow C$ is said to be nonexpansive [31-38] if $\|T x-T y\| \leq\|x-y\|$ for all $x, y \in C$. We use Fix $(T)$ to denote the set of fixed points of $T$. $T: C \rightarrow C$ is called a firmly nonexpansive mapping if, for all $x, y \in C,\|T x-T y\|^{2} \leq\langle x-y, T x-T y\rangle$. It is known that $T$ is firmly nonexpansive if and only if a mapping $2 T-I$ is nonexpansive, where $I$ is the identity mapping on $H$. T:C $\rightarrow H$ is said to be $L$-Lipschitz continuous if there exists a constant $L>0$ such that $\|T x-T y\| \leq L\|x-y\|$ for all $x, y \in C$. In such a case, $T$ is said to be $L$-Lipschitz continuous. Given a nonempty, closed convex subset $C$ of $H$, the mapping that assigns every point $x \in H$ to its unique nearest point in $C$ is called a metric projection onto $C$ and denoted by $P_{C}$, that is, $P_{C} x \in C$ and $\left\|x-P_{C} x\right\|=\inf \{\|x-y\|: y \in C\}$. The metric projection $P_{C}$ is a typical firmly nonexpansive mapping. The characteristic inequality of the projection is $\left\langle x-P_{C} x, y-P_{C} x\right\rangle \leq 0$ for all $x \in H, y \in C$.

\subsection{Equilibrium problem}

In this paper, we consider the split problem (1.1). In the sequel, we assume that the solution set $S$ of (1.1) is nonempty.

Problem 2.1 Assume that

(A1) $B: C \rightarrow H$ is an $\alpha$-inverse strongly $\psi$-monotone mapping; 
(A2) $\psi: C \rightarrow C$ is a weakly continuous and $\gamma$-strongly monotone mapping such that $R(\psi)=C$

(A3) $F: C \times C \rightarrow R$ is a bifunction;

(A4) $A: C \rightarrow H$ is a $\beta$-inverse-strongly monotone mapping.

Our objective is to

find $x^{*} \in \mathrm{GVI}(B, \psi, C)$ such that $\psi\left(x^{*}\right) \in \mathrm{EP}(F, A)$,

where $F$ satisfies the following conditions:

(F1) $F(x, x)=0$ for all $x \in C$;

(F2) $F$ is monotone, i.e., $F(x, y)+F(y, x) \leq 0$ for all $x, y \in C$;

(F3) for each $x, y, z \in C, \lim _{t \downarrow 0} F(t z+(1-t) x, y) \leq F(x, y)$;

(F4) for each $x \in C, y \mapsto F(x, y)$ is convex and lower semicontinuous.

In order to solve Problem 2.1, we need the following useful lemmas.

\subsection{Useful lemmas}

The following three lemmas are important tools for our main results in the next section. Note that these lemmas are used extensively in the literature.

Lemma 2.2 (Combettes and Hirstoaga's lemma [26]) Let $C$ be a nonempty closed convex subset of a real Hilbert space H. Let $F: C \times C \rightarrow R$ be a bifunction which satisfies conditions (F1)-(F4). Let $\lambda>0$ and $x \in C$. Then there exists $z \in C$ such that

$$
F(z, y)+\frac{1}{\lambda}\langle y-z, z-x\rangle \geq 0, \quad \forall y \in C .
$$

Further, if $T_{\lambda}(x)=\left\{z \in C: F(z, y)+\frac{1}{\lambda}\langle y-z, z-x\rangle \geq 0\right.$ for all $\left.y \in C\right\}$, then the following hold:

(a) $T_{\lambda}$ is single-valued and $T_{\lambda}$ is firmly nonexpansive;

(b) $\mathrm{EP}(F)$ is closed and convex and $\mathrm{EP}(F)=\operatorname{Fix}\left(T_{\lambda}\right)$.

Lemma 2.3 (Suzuki's lemma [39]) Let $\left\{x_{n}\right\}$ and $\left\{y_{n}\right\}$ be bounded sequences in a Banach space $X$ and let $\left\{\beta_{n}\right\}$ be a sequence in $[0,1]$ with $0<\liminf _{n \rightarrow \infty} \beta_{n} \leq \limsup _{n \rightarrow \infty} \beta_{n}<1$. Suppose $x_{n+1}=\left(1-\beta_{n}\right) y_{n}+\beta_{n} x_{n}$ for all $n \geq 0$ and $\lim \sup _{n \rightarrow \infty}\left(\left\|y_{n+1}-y_{n}\right\|-\left\|x_{n+1}-x_{n}\right\|\right) \leq 0$. Then $\lim _{n \rightarrow \infty}\left\|y_{n}-x_{n}\right\|=0$.

Lemma 2.4 (Xu's lemma [40]) Assume that $\left\{a_{n}\right\}$ is a sequence of nonnegative real numbers such that $a_{n+1} \leq\left(1-\gamma_{n}\right) a_{n}+\delta_{n} \gamma_{n}$, where $\left\{\gamma_{n}\right\}$ is a sequence in $(0,1)$ and $\left\{\delta_{n}\right\}$ is a sequence such that $\sum_{n=1}^{\infty} \gamma_{n}=\infty$ and $\lim \sup _{n \rightarrow \infty} \delta_{n} \leq 0\left(\right.$ or $\left.\sum_{n=1}^{\infty}\left|\delta_{n} \gamma_{n}\right|<\infty\right)$. Then $\lim _{n \rightarrow \infty} a_{n}=0$.

\section{Algorithms and convergence analysis}

In this section, we first present our algorithm for solving Problem 2.1. Assume that the conditions in Problem 2.1 are all satisfied.

Algorithm 3.1 Let $C$ be a nonempty closed and convex subset of a real Hilbert space $H$.

Step 0. (Initialization)

$$
x_{0} \in C
$$


Step 1. (Projection step) For $\left\{x_{n}\right\}$, let the sequence $\left\{u_{n}\right\}$ be generated iteratively by

$$
u_{n}=P_{C}\left[\alpha_{n} \delta \varphi\left(x_{n}\right)+\left(1-\alpha_{n}\right)\left(\psi\left(x_{n}\right)-\mu_{n} B x_{n}\right)\right], \quad n \geq 0,
$$

where $P_{C}$ is the metric projection, $\left\{\alpha_{n}\right\} \subset[0.1]$ is a real number sequence, $\varphi: C \rightarrow H$ is an $L$-Lipschitz continuous mapping and $\delta>0$ is a constant.

Step 2. (Proximal step) Find $\left\{z_{n}\right\}$ such that

$$
F\left(z_{n}, y\right)+\left\langle A u_{n}, y-z_{n}\right\rangle+\frac{1}{\lambda_{n}}\left\langle y-z_{n}, z_{n}-u_{n}\right\rangle \geq 0, \quad \forall y \in C,
$$

where $\left\{\lambda_{n}\right\} \subset(0, \infty)$ is a real number sequence.

Step 3. (Affine step) For the above sequences $\left\{x_{n}\right\}$ and $\left\{z_{n}\right\}$, let the $(n+1)$ th sequence $\left\{x_{n+1}\right\}$ be generated by

$$
\psi\left(x_{n+1}\right)=\beta_{n} \psi\left(x_{n}\right)+\left(1-\beta_{n}\right) z_{n}, \quad n \geq 0,
$$

where $\left\{\beta_{n}\right\} \subset[0,1]$ is a real number sequence.

Theorem 3.2 Suppose $S \neq \emptyset$. Assume that the following restrictions are satisfied:

(C1) $\lambda_{n} \in(a, b) \subset(0,2 \beta), \mu_{n} \in(c, d) \subset(0,2 \alpha)$ and $\gamma \in(L \delta, 2 \alpha)$;

(C2) $\lim _{n \rightarrow \infty}\left(\mu_{n+1}-\mu_{n}\right)=0$ and $\lim _{n \rightarrow \infty}\left(\lambda_{n+1}-\lambda_{n}\right)=0$;

(C3) $\lim _{n \rightarrow \infty} \alpha_{n}=0$ and $\sum_{n} \alpha_{n}=\infty$;

(C4) $\beta_{n} \in\left[\xi_{1}, \xi_{2}\right] \subset(0,1)$.

Then the sequence $\left\{x_{n}\right\}$ generated by Algorithm 3.1 converges strongly to $x^{*} \in S$, which solves the following variational inequality:

$$
\left\langle\delta \varphi\left(x^{*}\right)-\psi\left(x^{*}\right), \psi(x)-\psi\left(x^{*}\right)\right\rangle \leq 0, \quad \forall x \in \Omega
$$

Remark 3.3 The solution of variational inequality (3.1) is unique. As a matter of fact, if $\tilde{x} \in S$ also solves (3.1), we have

$$
\left\langle\delta \varphi\left(x^{*}\right)-\psi\left(x^{*}\right), \psi(\tilde{x})-\psi\left(x^{*}\right)\right\rangle \leq 0 \quad \text { and } \quad\left\langle\delta \varphi(\tilde{x})-\psi(\tilde{x}), \psi\left(x^{*}\right)-\psi(\tilde{x})\right\rangle \leq 0 .
$$

Adding up the above two inequalities, we deduce

$$
\left\langle\delta \varphi(\tilde{x})-\psi(\tilde{x})-\delta \varphi\left(x^{*}\right)+\psi\left(x^{*}\right), \psi\left(x^{*}\right)-\psi(\tilde{x})\right\rangle \leq 0 .
$$

It follows that

$$
\begin{aligned}
\left\|\psi\left(x^{*}\right)-\psi(\tilde{x})\right\|^{2} & \leq \delta\left\langle\varphi\left(x^{*}\right)-\varphi(\tilde{x}), \psi\left(x^{*}\right)-\psi(\tilde{x})\right\rangle \\
& \leq \delta\left\|\varphi\left(x^{*}\right)-\varphi(\tilde{x})\right\|\left\|\psi\left(x^{*}\right)-\psi(\tilde{x})\right\|,
\end{aligned}
$$

which implies that

$$
\left\|\psi\left(x^{*}\right)-\psi(\tilde{x})\right\| \leq \delta\left\|\varphi\left(x^{*}\right)-\varphi(\tilde{x})\right\| .
$$


Since $\psi$ is $\gamma$-strongly monotone, we have

$$
\gamma\left\|x^{*}-\tilde{x}\right\|^{2} \leq\left\langle\psi\left(x^{*}\right)-\psi(\tilde{x}), x^{*}-\tilde{x}\right\rangle \leq\left\|\psi\left(x^{*}\right)-\psi(\tilde{x})\right\|\left\|x^{*}-\tilde{x}\right\| .
$$

Hence,

$$
\gamma\left\|x^{*}-\tilde{x}\right\| \leq\left\|\psi\left(x^{*}\right)-\psi(\tilde{x})\right\| \leq \delta\left\|\varphi\left(x^{*}\right)-\varphi(\tilde{x})\right\| \leq \delta L\left\|x^{*}-\tilde{x}\right\| .
$$

This deduces the contraction because of $\delta L<\gamma$ by the assumption. Therefore, $x^{*}=\tilde{x}$. So, the solution of variational inequality (3.1) is unique.

Remark 3.4 Using the characteristic inequality of the projection, we have

$$
\breve{x} \in \operatorname{GVI}(B, \psi, C) \quad \Longleftrightarrow \quad \psi(\breve{x})=P_{C}(\psi(\breve{x})-v B \breve{x}), \quad \forall v>0 .
$$

\section{Remark 3.5}

$$
\|(\psi(x)-\mu B x)-(\psi(y)-\mu B y)\|^{2} \leq\|\psi(x)-\psi(y)\|^{2}+\mu(\mu-2 \alpha)\|B x-B y\|^{2} .
$$

In fact,

$$
\begin{aligned}
& \|(\psi(x)-\mu B x)-(\psi(y)-\mu B y)\|^{2} \\
& \quad=\|\psi(x)-\psi(y)\|^{2}-2 \mu\langle B x-B y, \psi(x)-\psi(y)\rangle+\mu^{2}\|B x-B y\|^{2} \\
& \quad \leq\|\psi(x)-\psi(y)\|^{2}-2 \mu \alpha\|B x-B y\|^{2}+\mu^{2}\|B x-B y\|^{2} \\
& \quad \leq\|\psi(x)-\psi(y)\|^{2}+\mu(\mu-2 \alpha)\|B x-B y\|^{2} .
\end{aligned}
$$

Next, we prove Theorem 3.2.

Proof Let $x^{\ddagger} \in \Omega$. Hence $x^{\ddagger} \in \operatorname{GVI}(B, \psi, C)$ and $\psi\left(x^{\ddagger}\right) \in \operatorname{EP}(F, A)$. Since $\mu_{n}>0$, from Remark 3.4 we have $\psi\left(x^{\ddagger}\right)=P_{C}\left[\psi\left(x^{\ddagger}\right)-\mu_{n} B x^{\ddagger}\right]$ for all $n \geq 0$. Thus,

$$
\begin{aligned}
\left\|u_{n}-\psi\left(x^{\ddagger}\right)\right\|= & \left\|P_{C}\left[\alpha_{n} \delta \varphi\left(x_{n}\right)+\left(1-\alpha_{n}\right)\left(\psi\left(x_{n}\right)-\mu_{n} B x_{n}\right)\right]-P_{C}\left[\psi\left(x^{\ddagger}\right)-\mu_{n} B x^{\ddagger}\right]\right\| \\
\leq & \| \alpha_{n}\left(\delta \varphi\left(x_{n}\right)-\psi\left(x^{\ddagger}\right)+\mu_{n} B x^{\ddagger}\right) \\
& +\left(1-\alpha_{n}\right)\left(\left(\psi\left(x_{n}\right)-\mu_{n} B x_{n}\right)-\left(\psi\left(x^{\ddagger}\right)-\mu_{n} B x^{\ddagger}\right)\right) \| \\
\leq & \alpha_{n}\left\|\delta \varphi\left(x_{n}\right)-\delta \varphi\left(x^{\ddagger}\right)\right\|+\alpha_{n}\left\|\delta \varphi\left(x^{\ddagger}\right)-\psi\left(x^{\ddagger}\right)+\mu_{n} B x^{\ddagger}\right\| \\
& +\left(1-\alpha_{n}\right)\left\|\left(\psi\left(x_{n}\right)-\mu_{n} B x_{n}\right)-\left(\psi\left(x^{\ddagger}\right)-\mu_{n} B x^{\ddagger}\right)\right\| \\
\leq & \alpha_{n} \delta L\left\|x_{n}-x^{\ddagger}\right\|+\alpha_{n}\left\|\delta \varphi\left(x^{\ddagger}\right)-\psi\left(x^{\ddagger}\right)+\mu_{n} B x^{\ddagger}\right\| \\
& +\left(1-\alpha_{n}\right)\left\|\psi\left(x_{n}\right)-\psi\left(x^{\ddagger}\right)\right\| \\
\leq & \alpha_{n} \delta L / \gamma\left\|\psi\left(x_{n}\right)-\psi\left(x^{\ddagger}\right)\right\|+\alpha_{n}\left\|\delta \varphi\left(x^{\ddagger}\right)-\psi\left(x^{\ddagger}\right)+\mu_{n} B x^{\ddagger}\right\| \\
& +\left(1-\alpha_{n}\right)\left\|\psi\left(x_{n}\right)-\psi\left(x^{\ddagger}\right)\right\| \\
= & {\left[1-(1-\delta L / \gamma) \alpha_{n}\right]\left\|\psi\left(x_{n}\right)-\psi\left(x^{\ddagger}\right)\right\|+\alpha_{n}\left\|\delta \varphi\left(x^{\ddagger}\right)-\psi\left(x^{\ddagger}\right)+\mu_{n} B x^{\ddagger}\right\| }
\end{aligned}
$$




$$
\begin{aligned}
\leq & {\left[1-(1-\delta L / \gamma) \alpha_{n}\right]\left\|\psi\left(x_{n}\right)-\psi\left(x^{\ddagger}\right)\right\| } \\
& +\alpha_{n}\left(\left\|\delta \varphi\left(x^{\ddagger}\right)-\psi\left(x^{\ddagger}\right)\right\|+2 \alpha\left\|B x^{\ddagger}\right\|\right) .
\end{aligned}
$$

By Algorithm 3.1, we have $z_{n}=T_{\lambda_{n}}\left(I-\lambda_{n} A\right) u_{n}$ for all $n \geq 0$. Noting that $\psi\left(x^{\ddagger}\right) \in \operatorname{EP}(F, A)$, we deduce $\psi\left(x^{\ddagger}\right)=T_{\lambda_{n}}\left(I-\lambda_{n} A\right) \psi\left(x^{\ddagger}\right)$ for all $n \geq 0$. It follows that

$$
\begin{aligned}
\left\|\psi\left(x_{n+1}\right)-\psi\left(x^{\ddagger}\right)\right\| \leq & \beta_{n}\left\|\psi\left(x_{n}\right)-\psi\left(x^{\ddagger}\right)\right\| \\
& +\left(1-\beta_{n}\right)\left\|T_{\lambda_{n}}\left(I-\lambda_{n} A\right) u_{n}-T_{\lambda_{n}}\left(I-\lambda_{n} A\right) \psi\left(x^{\ddagger}\right)\right\| \\
\leq & \beta_{n}\left\|\psi\left(x_{n}\right)-\psi\left(x^{\ddagger}\right)\right\|+\left(1-\beta_{n}\right)\left\|u_{n}-\psi\left(x^{\ddagger}\right)\right\| \\
\leq & \beta_{n}\left\|\psi\left(x_{n}\right)-\psi\left(x^{\ddagger}\right)\right\| \\
& +\left(1-\beta_{n}\right)\left[1-(1-\delta L / \gamma) \alpha_{n}\right]\left\|\psi\left(x_{n}\right)-\psi\left(x^{\ddagger}\right)\right\| \\
& +\left(1-\beta_{n}\right) \alpha_{n}\left(\left\|\delta \varphi\left(x^{\ddagger}\right)-\psi\left(x^{\ddagger}\right)\right\|+2 \alpha\left\|B x^{\ddagger}\right\|\right) \\
= & {\left[1-(1-\delta L / \gamma)\left(1-\beta_{n}\right) \alpha_{n}\right]\left\|\psi\left(x_{n}\right)-\psi\left(x^{\ddagger}\right)\right\| } \\
& +(1-\delta L / \gamma)\left(1-\beta_{n}\right) \alpha_{n} \frac{\left\|\delta \varphi\left(x^{\ddagger}\right)-\psi\left(x^{\ddagger}\right)\right\|+2 \alpha\left\|B x^{\ddagger}\right\| .}{1-\delta L / \gamma} .
\end{aligned}
$$

By induction

$$
\left\|\psi\left(x_{n}\right)-\psi\left(x^{\ddagger}\right)\right\| \leq \max \left\{\left\|\psi\left(x_{0}\right)-\psi\left(x^{\ddagger}\right)\right\|, \frac{\left\|\delta \varphi\left(x^{\ddagger}\right)-\psi\left(x^{\ddagger}\right)\right\|+2 \alpha\left\|B x^{\ddagger}\right\|}{1-\delta L / \gamma}\right\} .
$$

Hence, $\left\{\psi\left(x_{n}\right)\right\}$ is bounded. Since $\psi$ is $\gamma$-strongly monotone, we can get (by a similar technique as that in Remark 3.3) $\gamma\left\|x_{n}-x^{\ddagger}\right\| \leq\left\|\psi\left(x_{n}\right)-\psi\left(x^{\ddagger}\right)\right\|$. So, $\left\|x_{n}-x^{\ddagger}\right\| \leq$ $\frac{1}{\gamma}\left\|\psi\left(x_{n}\right)-\psi\left(x^{\ddagger}\right)\right\| \leq \frac{1}{\gamma} \max \left\{\left\|\psi\left(x_{0}\right)-\psi\left(x^{\ddagger}\right)\right\|, \frac{\left\|\delta \varphi\left(x^{\ddagger}\right)-\psi\left(x^{\ddagger}\right)\right\|+2 \alpha\left\|B x^{\ddagger}\right\|}{1-\delta L / \gamma}\right\}$. This implies that $\left\{x_{n}\right\}$ is bounded. Next, we show $\left\|x_{n+1}-x_{n}\right\| \rightarrow 0$. From Step 2 in Algorithm 3.1, we have

$$
F\left(z_{n}, y\right)+\frac{1}{\lambda_{n}}\left\langle y-z_{n}, z_{n}-\left(u_{n}-\lambda_{n} A u_{n}\right)\right\rangle \geq 0, \quad \forall y \in C .
$$

Taking $y=z_{n+1}$, we get

$$
F\left(z_{n}, z_{n+1}\right)+\frac{1}{\lambda_{n}}\left\langle z_{n+1}-z_{n}, z_{n}-\left(u_{n}-\lambda_{n} A u_{n}\right)\right| \geq 0 .
$$

Similarly, we also have

$$
F\left(z_{n+1}, z_{n}\right)+\frac{1}{\lambda_{n+1}}\left\langle z_{n}-z_{n+1}, z_{n+1}-\left(u_{n+1}-\lambda_{n+1} A u_{n+1}\right)\right\rangle \geq 0 .
$$

Adding up the above two inequalities, we get

$$
F\left(z_{n}, z_{n+1}\right)+F\left(z_{n+1}, z_{n}\right)+\left\langle A u_{n}-A u_{n+1}, z_{n+1}-z_{n}\right\rangle+\left\langle z_{n+1}-z_{n}, \frac{z_{n}-u_{n}}{\lambda_{n}}-\frac{z_{n+1}-u_{n+1}}{\lambda_{n+1}}\right\rangle \geq 0 .
$$

By the monotonicity of $F$, we have

$$
F\left(z_{n}, z_{n+1}\right)+F\left(z_{n+1}, z_{n}\right) \leq 0 .
$$


So,

$$
\left\langle A u_{n}-A u_{n+1}, z_{n+1}-z_{n}\right\rangle+\left\langle z_{n+1}-z_{n}, \frac{z_{n}-u_{n}}{\lambda_{n}}-\frac{z_{n+1}-u_{n+1}}{\lambda_{n+1}}\right\rangle \geq 0
$$

Thus,

$$
\lambda_{n}\left\langle A u_{n}-A u_{n+1}, z_{n+1}-z_{n}\right\rangle+\left\langle z_{n+1}-z_{n}, z_{n}-z_{n+1}+z_{n+1}-u_{n}-\frac{\lambda_{n}}{\lambda_{n+1}}\left(z_{n+1}-u_{n+1}\right)\right\rangle \geq 0 .
$$

It follows that

$$
\begin{aligned}
\left\|z_{n+1}-z_{n}\right\|^{2} \leq & \lambda_{n}\left\langle A u_{n}-A u_{n+1}, z_{n+1}-z_{n}\right\rangle \\
& +\left\langle z_{n+1}-z_{n}, u_{n+1}-u_{n}+\left(1-\frac{\lambda_{n}}{\lambda_{n+1}}\right)\left(z_{n+1}-u_{n+1}\right)\right\rangle \\
= & \left\langle\left(I-\lambda_{n} A\right) u_{n+1}-\left(I-\lambda_{n} A\right) u_{n}, z_{n+1}-z_{n}\right\rangle \\
& +\left\langle z_{n+1}-z_{n},\left(1-\frac{\lambda_{n}}{\lambda_{n+1}}\right)\left(z_{n+1}-u_{n+1}\right)\right\rangle \\
\leq & \left\|\left(I-\lambda_{n} A\right) u_{n+1}-\left(I-\lambda_{n} A\right) u_{n}\right\|\left\|z_{n+1}-z_{n}\right\| \\
& +\left|1-\frac{\lambda_{n}}{\lambda_{n+1}}\right|\left\|z_{n+1}-z_{n}\right\|\left\|z_{n+1}-u_{n+1}\right\| \\
\leq & \left\|z_{n+1}-z_{n}\right\|\left(\left\|u_{n+1}-u_{n}\right\|+\left|1-\frac{\lambda_{n}}{\lambda_{n+1}}\right|\left\|z_{n+1}-u_{n+1}\right\|\right)
\end{aligned}
$$

and hence

$$
\begin{aligned}
\left\|z_{n+1}-z_{n}\right\| & \leq\left\|u_{n+1}-u_{n}\right\|+\left|1-\frac{\lambda_{n}}{\lambda_{n+1}}\right|\left\|z_{n+1}-u_{n+1}\right\| \\
& \leq\left\|u_{n+1}-u_{n}\right\|+\frac{1}{a}\left|\lambda_{n+1}-\lambda_{n}\right|\left\|z_{n+1}-u_{n+1}\right\| .
\end{aligned}
$$

By Algorithm 3.1, we have

$$
\begin{aligned}
\left\|u_{n+1}-u_{n}\right\|= & \| P_{C}\left[\alpha_{n+1} \delta \varphi\left(x_{n+1}\right)+\left(1-\alpha_{n+1}\right)\left(\psi\left(x_{n+1}\right)-\mu_{n+1} B x_{n+1}\right)\right] \\
& -P_{C}\left[\alpha_{n} \delta \varphi\left(x_{n}\right)+\left(1-\alpha_{n}\right)\left(\psi\left(x_{n}\right)-\mu_{n} B x_{n}\right)\right] \| \\
\leq & \|\left[\alpha_{n+1} \delta \varphi\left(x_{n+1}\right)+\left(1-\alpha_{n+1}\right)\left(\psi\left(x_{n+1}\right)-\mu_{n+1} B x_{n+1}\right)\right] \\
& -\left[\alpha_{n} \delta \varphi\left(x_{n}\right)+\left(1-\alpha_{n}\right)\left(\psi\left(x_{n}\right)-\mu_{n} B x_{n}\right)\right] \| \\
\leq & \alpha_{n+1} \delta\left\|\varphi\left(x_{n+1}\right)-\varphi\left(x_{n}\right)\right\|+\delta\left|\alpha_{n+1}-\alpha_{n}\right|\left\|\varphi\left(x_{n}\right)\right\| \\
& +\left(1-\alpha_{n+1}\right)\left\|\psi\left(x_{n+1}\right)-\mu_{n+1} B x_{n+1}-\left(\psi\left(x_{n}\right)-\mu_{n+1} B x_{n}\right)\right\| \\
& +\left|\alpha_{n+1}-\alpha_{n}\right|\left\|\psi\left(x_{n}\right)\right\|+\left|\mu_{n+1}-\mu_{n}\right|\left\|B\left(x_{n}\right)\right\|+\left|\alpha_{n+1} \mu_{n+1}-\alpha_{n} \mu_{n}\right|\left\|B\left(x_{n}\right)\right\| \\
\leq & \alpha_{n+1} \delta L\left\|x_{n+1}-x_{n}\right\|+\left(1-\alpha_{n+1}\right)\left\|\psi\left(x_{n+1}\right)-\psi\left(x_{n}\right)\right\| \\
& +\left|\alpha_{n+1}-\alpha_{n}\right|\left(\delta\left\|\varphi\left(x_{n}\right)\right\|+\left\|\psi\left(x_{n}\right)\right\|\right)+\left|\mu_{n+1}-\mu_{n}\right|\left\|B\left(x_{n}\right)\right\| \\
& +\left|\alpha_{n+1} \mu_{n+1}-\alpha_{n} \mu_{n}\right|\left\|B\left(x_{n}\right)\right\|
\end{aligned}
$$




$$
\begin{aligned}
\leq & \alpha_{n+1}(\delta L / \gamma)\left\|\psi\left(x_{n+1}\right)-\psi\left(x_{n}\right)\right\|+\left(1-\alpha_{n+1}\right)\left\|\psi\left(x_{n+1}\right)-\psi\left(x_{n}\right)\right\| \\
& +\left|\alpha_{n+1}-\alpha_{n}\right|\left(\delta\left\|\varphi\left(x_{n}\right)\right\|+\left\|\psi\left(x_{n}\right)\right\|\right)+\left|\mu_{n+1}-\mu_{n}\right|\left\|B\left(x_{n}\right)\right\| \\
& +\left|\alpha_{n+1} \mu_{n+1}-\alpha_{n} \mu_{n}\right|\left\|B\left(x_{n}\right)\right\| \\
= & {\left[1-(1-\delta L / \gamma) \alpha_{n+1}\right]\left\|\psi\left(x_{n+1}\right)-\psi\left(x_{n}\right)\right\| } \\
& +\left|\alpha_{n+1}-\alpha_{n}\right|\left(\delta\left\|\varphi\left(x_{n}\right)\right\|+\left\|\psi\left(x_{n}\right)\right\|\right) \\
& +\left|\mu_{n+1}-\mu_{n}\right|\left\|B\left(x_{n}\right)\right\|+\left|\alpha_{n+1} \mu_{n+1}-\alpha_{n} \mu_{n}\right|\left\|B\left(x_{n}\right)\right\| .
\end{aligned}
$$

Therefore,

$$
\begin{aligned}
\left\|z_{n+1}-z_{n}\right\| \leq & {\left[1-(1-\delta L / \gamma) \alpha_{n+1}\right]\left\|\psi\left(x_{n+1}\right)-\psi\left(x_{n}\right)\right\| } \\
& +\left|\alpha_{n+1}-\alpha_{n}\right|\left(\delta\left\|\varphi\left(x_{n}\right)\right\|+\left\|\psi\left(x_{n}\right)\right\|\right) \\
& +\left|\mu_{n+1}-\mu_{n}\right|\left\|B\left(x_{n}\right)\right\|+\left|\alpha_{n+1} \mu_{n+1}-\alpha_{n} \mu_{n}\right|\left\|B\left(x_{n}\right)\right\| \\
& +\frac{1}{a}\left|\lambda_{n+1}-\lambda_{n}\right|\left\|z_{n+1}-u_{n+1}\right\| .
\end{aligned}
$$

It follows that

$$
\begin{aligned}
\left\|z_{n+1}-z_{n}\right\|-\left\|\psi\left(x_{n+1}\right)-\psi\left(x_{n}\right)\right\| \leq & \left|\alpha_{n+1}-\alpha_{n}\right|\left(\delta\left\|\varphi\left(x_{n}\right)\right\|+\left\|\psi\left(x_{n}\right)\right\|\right) \\
& +\left|\mu_{n+1}-\mu_{n}\right|\left\|B\left(x_{n}\right)\right\|+\left|\alpha_{n+1} \mu_{n+1}-\alpha_{n} \mu_{n}\right|\left\|B\left(x_{n}\right)\right\| \\
& +\frac{1}{a}\left|\lambda_{n+1}-\lambda_{n}\right|\left\|z_{n+1}-u_{n+1}\right\| .
\end{aligned}
$$

Since $\lim _{n \rightarrow \infty} \alpha_{n}=0, \lim _{n \rightarrow \infty}\left(\mu_{n+1}-\mu_{n}\right)=0, \lim _{n \rightarrow \infty}\left(\lambda_{n+1}-\lambda_{n}\right)=0$ and the sequences $\left\{\varphi\left(x_{n}\right)\right\},\left\{\psi\left(x_{n}\right)\right\},\left\{z_{n}\right\},\left\{u_{n}\right\}$ and $\left\{B x_{n}\right\}$ are bounded, we have

$$
\limsup _{n \rightarrow \infty}\left(\left\|z_{n+1}-z_{n}\right\|-\left\|\psi\left(x_{n+1}\right)-\psi\left(x_{n}\right)\right\|\right) \leq 0
$$

By Lemma 2.3, we obtain

$$
\lim _{n \rightarrow \infty}\left\|z_{n}-\psi\left(x_{n}\right)\right\|=0
$$

Hence,

$$
\lim _{n \rightarrow \infty}\left\|\psi\left(x_{n+1}\right)-\psi\left(x_{n}\right)\right\|=\lim _{n \rightarrow \infty}\left(1-\beta_{n}\right)\left\|z_{n}-\psi\left(x_{n}\right)\right\|=0 .
$$

This together with the $\gamma$-strong monotonicity of $\psi$ implies that

$$
\lim _{n \rightarrow \infty}\left\|x_{n+1}-x_{n}\right\|=0 .
$$

By the convexity of the norm, we have

$$
\begin{aligned}
& \left\|\psi\left(x_{n+1}\right)-\psi\left(x^{\ddagger}\right)\right\|^{2} \\
& \quad=\left\|\beta_{n}\left(\psi\left(x_{n}\right)-\psi\left(x^{\ddagger}\right)\right)+\left(1-\beta_{n}\right)\left(z_{n}-\psi\left(x^{\ddagger}\right)\right)\right\|^{2}
\end{aligned}
$$




$$
\begin{aligned}
\leq & \beta_{n}\left\|\psi\left(x_{n}\right)-\psi\left(x^{\ddagger}\right)\right\|^{2}+\left(1-\beta_{n}\right)\left\|z_{n}-\psi\left(x^{\ddagger}\right)\right\|^{2} \\
\leq & \beta_{n}\left\|\psi\left(x_{n}\right)-\psi\left(x^{\ddagger}\right)\right\|^{2}+\left(1-\beta_{n}\right)\left[\| \alpha_{n}\left(\delta \varphi\left(x_{n}\right)-\psi\left(x^{\ddagger}\right)+\mu_{n} B x^{\ddagger}\right)\right. \\
& \left.+\left(1-\alpha_{n}\right)\left(\left(\psi\left(x_{n}\right)-\mu_{n} B x_{n}\right)-\left(\psi\left(x^{\ddagger}\right)-\mu_{n} B x^{\ddagger}\right)\right) \|\right]^{2} \\
\leq & \beta_{n}\left\|\psi\left(x_{n}\right)-\psi\left(x^{\ddagger}\right)\right\|^{2}+\left(1-\beta_{n}\right)\left[\alpha_{n}\left\|\delta \varphi\left(x_{n}\right)-\psi\left(x^{\ddagger}\right)+\mu_{n} B x^{\ddagger}\right\|^{2}\right. \\
& +\left(1-\alpha_{n}\right)\left\|\left(\psi\left(x_{n}\right)-\mu_{n} B x_{n}\right)-\left(\psi\left(x^{\ddagger}\right)-\mu_{n} B x^{\ddagger}\right)\right\|^{2} \\
& \left.+2 \alpha_{n}\left(1-\alpha_{n}\right)\left\|\delta \varphi\left(x_{n}\right)-\psi\left(x^{\ddagger}\right)+\mu_{n} B x^{\ddagger}\right\|\left\|\left(\psi\left(x_{n}\right)-\mu_{n} B x_{n}\right)-\left(\psi\left(x^{\ddagger}\right)-\mu_{n} B x^{\ddagger}\right)\right\|\right] \\
\leq & \beta_{n}\left\|\psi\left(x_{n}\right)-\psi\left(x^{\ddagger}\right)\right\|^{2} \\
& +\left(1-\beta_{n}\right)\left(1-\alpha_{n}\right)\left\|\left(\psi\left(x_{n}\right)-\mu_{n} B x_{n}\right)-\left(\psi\left(x^{\ddagger}\right)-\mu_{n} B x^{\ddagger}\right)\right\|^{2}+\alpha_{n} M,
\end{aligned}
$$

where $M>0$ is some constant. From Remark 3.5, we derive

$$
\left\|\left(\psi\left(x_{n}\right)-\mu_{n} B x_{n}\right)-\left(\psi\left(x^{\ddagger}\right)-\mu_{n} B x^{\ddagger}\right)\right\|^{2} \leq\left\|\psi\left(x_{n}\right)-\psi\left(x^{\ddagger}\right)\right\|^{2}+\mu_{n}\left(\mu_{n}-2 \alpha\right)\left\|B x_{n}-B x^{\ddagger}\right\|^{2} .
$$

Thus,

$$
\begin{aligned}
\left\|\psi\left(x_{n+1}\right)-\psi\left(x^{\ddagger}\right)\right\|^{2} \leq & \beta_{n}\left\|\psi\left(x_{n}\right)-\psi\left(x^{\ddagger}\right)\right\|^{2}+\left(1-\beta_{n}\right)\left(1-\alpha_{n}\right)\left(\left\|\psi\left(x_{n}\right)-\psi\left(x^{\ddagger}\right)\right\|^{2}\right. \\
& \left.+\mu_{n}\left(\mu_{n}-2 \alpha\right)\left\|B x_{n}-B x^{\ddagger}\right\|^{2}\right)+\alpha_{n} M \\
\leq & \left\|\psi\left(x_{n}\right)-\psi\left(x^{\ddagger}\right)\right\|^{2} \\
& +\left(1-\beta_{n}\right)\left(1-\alpha_{n}\right) \mu_{n}\left(\mu_{n}-2 \alpha\right)\left\|B x_{n}-B x^{\ddagger}\right\|^{2}+\alpha_{n} M .
\end{aligned}
$$

So,

$$
\begin{aligned}
& \left(1-\beta_{n}\right)\left(1-\alpha_{n}\right) \mu_{n}\left(2 \alpha-\mu_{n}\right)\left\|B x_{n}-B x^{\ddagger}\right\|^{2} \\
& \quad \leq\left\|\psi\left(x_{n}\right)-\psi\left(x^{\ddagger}\right)\right\|^{2}-\left\|\psi\left(x_{n+1}\right)-\psi\left(x^{\ddagger}\right)\right\|^{2}+\alpha_{n} M \\
& \quad \leq\left(\left\|\psi\left(x_{n}\right)-\psi\left(x^{\ddagger}\right)\right\|+\left\|\psi\left(x_{n+1}\right)-\psi\left(x^{\ddagger}\right)\right\|\right)\left\|\psi\left(x_{n+1}\right)-\psi\left(x_{n}\right)\right\|+\alpha_{n} M .
\end{aligned}
$$

Since $\alpha_{n} \rightarrow 0,\left\|\psi\left(x_{n+1}\right)-\psi\left(x_{n}\right)\right\| \rightarrow 0$ and $\liminf _{n \rightarrow \infty}\left(1-\beta_{n}\right)\left(1-\alpha_{n}\right) \mu_{n}\left(2 \alpha-\mu_{n}\right)>0$, we obtain

$$
\lim _{n \rightarrow \infty}\left\|B x_{n}-B x^{\ddagger}\right\|=0 .
$$

Set $y_{n}=\psi\left(x_{n}\right)-\mu_{n} B x_{n}-\left(\psi\left(x^{\ddagger}\right)-\mu_{n} B x^{\ddagger}\right)$ for all $n$. By using the property of projection, we get

$$
\begin{aligned}
\left\|u_{n}-\psi\left(x^{\ddagger}\right)\right\|^{2}= & \left\|P_{C}\left[\alpha_{n} \delta \varphi\left(x_{n}\right)+\left(1-\alpha_{n}\right)\left(\psi\left(x_{n}\right)-\mu_{n} B x_{n}\right)\right]-P_{C}\left[\psi\left(x^{\ddagger}\right)-\mu_{n} B x^{\ddagger}\right]\right\|^{2} \\
\leq & \left\langle\alpha_{n}\left(\delta \varphi\left(x_{n}\right)-\psi\left(x^{\ddagger}\right)+\mu_{n} B x^{\ddagger}\right)+\left(1-\alpha_{n}\right) y_{n}, u_{n}-\psi\left(x^{\ddagger}\right)\right\rangle \\
= & \frac{1}{2}\left\{\left\|\alpha_{n}\left(\delta \varphi\left(x_{n}\right)-\psi\left(x^{\ddagger}\right)+\mu_{n} B x^{\ddagger}\right)+\left(1-\alpha_{n}\right) y_{n}\right\|^{2}+\left\|u_{n}-\psi\left(x^{\ddagger}\right)\right\|^{2}\right. \\
& \left.-\left\|\alpha_{n}\left(\delta \varphi\left(x_{n}\right)-\psi\left(x^{\ddagger}\right)+\mu_{n} B x^{\ddagger}\right)+\left(1-\alpha_{n}\right) y_{n}-u_{n}+\psi\left(x^{\ddagger}\right)\right\|^{2}\right\} \\
\leq & \frac{1}{2}\left\{\alpha_{n}\left\|\delta \varphi\left(x_{n}\right)-\psi\left(x^{\ddagger}\right)+\mu_{n} B x^{\ddagger}\right\|^{2}\right.
\end{aligned}
$$




$$
\begin{aligned}
& +\left(1-\alpha_{n}\right)\left\|\psi\left(x_{n}\right)-\psi\left(x^{\ddagger}\right)\right\|^{2}+\left\|u_{n}-\psi\left(x^{\ddagger}\right)\right\|^{2} \\
& -\| \alpha_{n}\left(\delta \varphi\left(x_{n}\right)-\psi\left(x^{\ddagger}\right)+\mu_{n} B x^{\ddagger}-y_{n}\right) \\
& \left.+\psi\left(x_{n}\right)-u_{n}-\mu_{n}\left(B x_{n}-B x^{\ddagger}\right) \|^{2}\right\} \\
= & \frac{1}{2}\left\{\alpha_{n}\left\|\delta \varphi\left(x_{n}\right)-\psi\left(x^{\ddagger}\right)+\mu_{n} B x^{\ddagger}\right\|^{2}\right. \\
& +\left(1-\alpha_{n}\right)\left\|\psi\left(x_{n}\right)-\psi\left(x^{\ddagger}\right)\right\|^{2}+\left\|u_{n}-\psi\left(x^{\ddagger}\right)\right\|^{2} \\
& -\left\|\psi\left(x_{n}\right)-u_{n}\right\|^{2}-\mu_{n}^{2}\left\|B x_{n}-B x^{\ddagger}\right\| \\
& -\alpha_{n}^{2}\left\|\delta \varphi\left(x_{n}\right)-\psi\left(x^{\ddagger}\right)+\mu_{n} B x^{\ddagger}-y_{n}\right\|^{2} \\
& +2 \mu_{n} \alpha_{n}\left\langle B x_{n}-B x^{\ddagger}, \delta \varphi\left(x_{n}\right)-\psi\left(x^{\ddagger}\right)+\mu_{n} B x^{\ddagger}-y_{n}\right\rangle \\
& +2 \mu_{n}\left\langle\psi\left(x_{n}\right)-u_{n}, B x_{n}-B x^{\ddagger}\right\rangle \\
& \left.-2 \alpha_{n}\left\langle\psi\left(x_{n}\right)-u_{n}, \delta \varphi\left(x_{n}\right)-\psi\left(x^{\ddagger}\right)+\mu_{n} B x^{\ddagger}-y_{n}\right\rangle\right\} .
\end{aligned}
$$

It follows that

$$
\begin{aligned}
\left\|u_{n}-\psi\left(x^{\ddagger}\right)\right\|^{2} \leq & \alpha_{n}\left\|\delta \varphi\left(x_{n}\right)-\psi\left(x^{\ddagger}\right)+\mu_{n} B x^{\ddagger}\right\|^{2} \\
& +\left(1-\alpha_{n}\right)\left\|\psi\left(x_{n}\right)-\psi\left(x^{\ddagger}\right)\right\|^{2}-\left\|\psi\left(x_{n}\right)-u_{n}\right\|^{2} \\
& +2 \mu_{n} \alpha_{n}\left\|B x_{n}-B x^{\ddagger}\right\|\left\|\delta \varphi\left(x_{n}\right)-\psi\left(x^{\ddagger}\right)+\mu_{n} B x^{\ddagger}-y_{n}\right\| \\
& +2 \mu_{n}\left\|\psi\left(x_{n}\right)-u_{n}\right\|\left\|B x_{n}-B x^{\ddagger}\right\| \\
& +2 \alpha_{n}\left\|\psi\left(x_{n}\right)-u_{n}\right\|\left\|\delta \varphi\left(x_{n}\right)-\psi\left(x^{\ddagger}\right)+\mu_{n} B x^{\ddagger}-y_{n}\right\| .
\end{aligned}
$$

From (3.3) and (3.5), we have

$$
\begin{aligned}
\| \psi( & \left.x_{n+1}\right)-\psi\left(x^{\ddagger}\right) \|^{2} \\
\leq & \beta_{n}\left\|\psi\left(x_{n}\right)-\psi\left(x^{\ddagger}\right)\right\|^{2}+\left(1-\beta_{n}\right)\left\|u_{n}-\psi\left(x^{\ddagger}\right)\right\|^{2} \\
\leq & \beta_{n}\left\|\psi\left(x_{n}\right)-\psi\left(x^{\ddagger}\right)\right\|^{2}+\left(1-\beta_{n}\right) \alpha_{n}\left\|\delta \varphi\left(x_{n}\right)-\psi\left(x^{\ddagger}\right)+\mu_{n} B x^{\ddagger}\right\|^{2} \\
& +\left(1-\alpha_{n}\right)\left(1-\beta_{n}\right)\left\|\psi\left(x_{n}\right)-\psi\left(x^{\ddagger}\right)\right\|^{2}-\left(1-\beta_{n}\right)\left\|\psi\left(x_{n}\right)-u_{n}\right\|^{2} \\
& +2 \mu_{n}\left(1-\beta_{n}\right) \alpha_{n}\left\|B x_{n}-B x^{\ddagger}\right\|\left\|\delta \varphi\left(x_{n}\right)-\psi\left(x^{\ddagger}\right)+\mu_{n} B x^{\ddagger}-y_{n}\right\| \\
& +2 \mu_{n}\left(1-\beta_{n}\right)\left\|\psi\left(x_{n}\right)-u_{n}\right\|\left\|B x_{n}-B x^{\ddagger}\right\| \\
& +2\left(1-\beta_{n}\right) \alpha_{n}\left\|\psi\left(x_{n}\right)-u_{n}\right\|\left\|\delta \varphi\left(x_{n}\right)-\psi\left(x^{\ddagger}\right)+\mu_{n} B x^{\ddagger}-y_{n}\right\| \\
\leq & \left\|\psi\left(x_{n}\right)-\psi\left(x^{\ddagger}\right)\right\|^{2}+\alpha_{n}\left\|\delta \varphi\left(x_{n}\right)-\psi\left(x^{\ddagger}\right)+\mu_{n} B x^{\ddagger}\right\|^{2} \\
& -\left(1-\beta_{n}\right)\left\|\psi\left(x_{n}\right)-u_{n}\right\|^{2} \\
& +2 \mu_{n} \alpha_{n}\left\|B x_{n}-B x^{\ddagger}\right\|\left\|\delta \varphi\left(x_{n}\right)-\psi\left(x^{\ddagger}\right)+\mu_{n} B x^{\ddagger}-y_{n}\right\| \\
& +2 \mu_{n}\left\|\psi\left(x_{n}\right)-u_{n}\right\|\left\|B x_{n}-B x^{\ddagger}\right\| \\
& +2 \alpha_{n}\left\|\psi\left(x_{n}\right)-u_{n}\right\|\left\|\delta \varphi\left(x_{n}\right)-\psi\left(x^{\ddagger}\right)+\mu_{n} B x^{\ddagger}-y_{n}\right\| .
\end{aligned}
$$


Then we obtain

$$
\begin{aligned}
\left(1-\beta_{n}\right)\left\|\psi\left(x_{n}\right)-u_{n}\right\|^{2} \leq & \left(\left\|\psi\left(x_{n}\right)-\psi\left(x^{\ddagger}\right)\right\|+\left\|\psi\left(x_{n+1}\right)-\psi\left(x^{\ddagger}\right)\right\|\right)\left\|\psi\left(x_{n+1}\right)-\psi\left(x_{n}\right)\right\| \\
& +\alpha_{n}\left\|\delta \varphi\left(x_{n}\right)-\psi\left(x^{\ddagger}\right)+\mu_{n} B x^{\ddagger}\right\|^{2} \\
& +2 \mu_{n} \alpha_{n}\left\|B x_{n}-B x^{\ddagger}\right\|\left\|\delta \varphi\left(x_{n}\right)-\psi\left(x^{\ddagger}\right)+\mu_{n} B x^{\ddagger}-y_{n}\right\| \\
& +2 \mu_{n}\left\|\psi\left(x_{n}\right)-u_{n}\right\|\left\|B x_{n}-B x^{\ddagger}\right\| \\
& +2 \alpha_{n}\left\|\psi\left(x_{n}\right)-u_{n}\right\|\left\|\delta \varphi\left(x_{n}\right)-\psi\left(x^{\ddagger}\right)+\mu_{n} B x^{\ddagger}-y_{n}\right\| .
\end{aligned}
$$

Since $\lim _{n \rightarrow \infty} \alpha_{n}=0, \lim _{n \rightarrow \infty}\left\|\psi\left(x_{n+1}\right)-\psi\left(x_{n}\right)\right\|=0$ and $\lim _{n \rightarrow \infty}\left\|B x_{n}-B x^{\ddagger}\right\|=0$, we have

$$
\lim _{n \rightarrow \infty}\left\|\psi\left(x_{n}\right)-u_{n}\right\|=0
$$

Next, we prove $\lim \sup _{n \rightarrow \infty}\left\langle\delta \varphi\left(x^{*}\right)-\psi\left(x^{*}\right), u_{n}-\psi\left(x^{*}\right)\right\rangle \leq 0$, where $x^{*}$ is the unique solution of (3.1). We take a subsequence $\left\{u_{n_{i}}\right\}$ of $\left\{u_{n}\right\}$ such that

$$
\begin{aligned}
& \limsup _{n \rightarrow \infty}\left\langle\delta \varphi\left(x^{*}\right)-\psi\left(x^{*}\right), u_{n}-\psi\left(x^{*}\right)\right\rangle \\
& \quad=\lim _{i \rightarrow \infty}\left\langle\delta \varphi\left(x^{*}\right)-\psi\left(x^{*}\right), u_{n_{i}}-\psi\left(x^{*}\right)\right\rangle \\
& \quad=\lim _{i \rightarrow \infty}\left\langle\delta \varphi\left(x^{*}\right)-\psi\left(x^{*}\right), \psi\left(x_{n_{i}}\right)-\psi\left(x^{*}\right)\right\rangle .
\end{aligned}
$$

Since $\left\{x_{n_{i}}\right\}$ is bounded, there exists a subsequence $\left\{x_{n_{i j}}\right\}$ of $\left\{x_{n_{i}}\right\}$ which converges weakly to some point $z \in C$. Without loss of generality, we may assume that $x_{n_{i}} \rightarrow z$. This implies that $\psi\left(x_{n_{i}}\right) \rightarrow \psi(z)$ due to the weak continuity of $\psi$. Now, we show $z \in S$. We firstly show $z \in \operatorname{EP}(F, A)$. Since $z_{n}=T_{\lambda_{n}}\left(u_{n}-\lambda_{n} A u_{n}\right)$, for any $y \in C$, we have

$$
F\left(z_{n}, y\right)+\frac{1}{\lambda_{n}}\left\langle y-z_{n}, z_{n}-\left(u_{n}-\lambda_{n} A u_{n}\right)\right| \geq 0 .
$$

From the monotonicity of $F$, we have

$$
\frac{1}{\lambda_{n}}\left\langle y-z_{n}, z_{n}-\left(u_{n}-\lambda_{n} A u_{n}\right)\right\rangle \geq F\left(y, z_{n}\right), \quad \forall y \in C .
$$

Hence,

$$
\left\langle y-z_{n_{i}}, \frac{z_{n_{i}}-u_{n_{i}}}{\lambda_{n_{i}}}+A u_{n_{i}}\right\rangle \geq F\left(y, z_{n_{i}}\right), \quad \forall y \in C .
$$

Put $v_{t}=t y+(1-t) z$ for all $t \in(0,1]$ and $y \in C$. Then we have $v_{t} \in C$. So, from (3.8) we have

$$
\begin{aligned}
\left\langle v_{t}-z_{n_{i}}, A v_{t}\right\rangle \geq & \left\langle v_{t}-z_{n_{i}}, A v_{t}\right\rangle-\left\langle v_{t}-z_{n_{i}}, \frac{z_{n_{i}}-u_{n_{i}}}{\lambda_{n_{i}}}+A u_{n_{i}}\right\rangle+F\left(v_{t}, z_{n_{i}}\right) \\
= & \left\langle v_{t}-z_{n_{i}}, A v_{t}-A z_{n_{i}}\right\rangle+\left\langle v_{t}-z_{n_{i}}, A z_{n_{i}}-A u_{n_{i}}\right\rangle \\
& -\left\langle v_{t}-z_{n_{i}}, \frac{z_{n_{i}}-u_{n_{i}}}{\lambda_{n_{i}}}\right\rangle+F\left(v_{t}, z_{n_{i}}\right) .
\end{aligned}
$$


Note that $\left\|A z_{n_{i}}-A u_{n_{i}}\right\| \leq \frac{1}{\beta}\left\|z_{n_{i}}-u_{n_{i}}\right\| \rightarrow 0$. Further, from the monotonicity of $A$, we have $\left\langle v_{t}-z_{n_{i}}, A v_{t}-A z_{n_{i}}\right\rangle \geq 0$. Letting $i \rightarrow \infty$ in (3.9), we have $\left\langle v_{t}-z, A v_{t}\right\rangle \geq F\left(v_{t}, z\right)$. This together with (F1), (F4) implies that

$$
\begin{aligned}
0 & =F\left(v_{t}, v_{t}\right) \leq t F\left(v_{t}, y\right)+(1-t) F\left(v_{t}, z\right) \\
& \leq t F\left(v_{t}, y\right)+(1-t)\left\langle v_{t}-z, A v_{t}\right\rangle \\
& =t F\left(v_{t}, y\right)+(1-t) t\left\langle y-z, A v_{t}\right\rangle,
\end{aligned}
$$

and hence $0 \leq F\left(v_{t}, y\right)+(1-t)\left\langle A v_{t}, y-z\right\rangle$. Letting $t \rightarrow 0$, we have $0 \leq F(z, y)+\langle y-z, A z\rangle$. This implies that $z \in \operatorname{EP}(F, A)$. Next, we only need to prove $z \in \operatorname{GVI}(B, \psi, C)$. Set

$$
R v= \begin{cases}B v+N_{C}(v), & v \in C, \\ \emptyset, & v \notin C .\end{cases}
$$

By [41], we know that $R$ is maximal $\psi$-monotone. Let $(v, w) \in G(R)$. Since $w-B v \in N_{C}(v)$ and $x_{n} \in C$, we have $\left\langle\psi(v)-\psi\left(x_{n}\right), w-B v\right\rangle \geq 0$. Noting that $u_{n}=P_{C}\left[\alpha_{n} \delta \varphi\left(x_{n}\right)+(1-\right.$ $\left.\left.\alpha_{n}\right)\left(\psi\left(x_{n}\right)-\mu_{n} B x_{n}\right)\right]$, we get

$$
\left\langle\psi(v)-u_{n}, u_{n}-\left[\alpha_{n} \delta \varphi\left(x_{n}\right)+\left(1-\alpha_{n}\right)\left(\psi\left(x_{n}\right)-\mu_{n} B x_{n}\right)\right]\right\rangle \geq 0 .
$$

It follows that

$$
\left\langle\psi(v)-u_{n}, \frac{u_{n}-\psi\left(x_{n}\right)}{\mu_{n}}+B x_{n}-\frac{\alpha_{n}}{\mu_{n}}\left(\delta \varphi\left(x_{n}\right)-\psi\left(x_{n}\right)+\mu_{n} B x_{n}\right)\right\rangle \geq 0 .
$$

Then

$$
\begin{aligned}
&\left\langle\psi(v)-\psi\left(x_{n_{i}}\right), w\right\rangle \geq\left\langle\psi(v)-\psi\left(x_{n_{i}}\right), B v\right\rangle \\
& \geq\left\langle\psi(v)-\psi\left(x_{n_{i}}\right), B v\right\rangle-\left\langle\psi(v)-u_{n_{i}}, \frac{u_{n_{i}}-\psi\left(x_{n_{i}}\right)}{\mu_{n_{i}}}\right\rangle \\
&-\left\langle\psi(v)-u_{n_{i}}, B x_{n_{i}}\right\rangle+\frac{\alpha_{n_{i}}}{\mu_{n_{i}}}\left\langle\psi(v)-u_{n_{i}}, \delta \varphi\left(x_{n_{i}}\right)-\psi\left(x_{n_{i}}\right)+\mu_{n_{i}} B x_{n_{i}}\right\rangle \\
&=\left\langle\psi(v)-\psi\left(x_{n_{i}}\right), B v-B x_{n_{i}}\right\rangle+\left\langle\psi(v)-\psi\left(x_{n_{i}}\right), B x_{n_{i}}\right\rangle \\
&-\left\langle\psi(v)-u_{n_{i}}, \frac{u_{n_{i}}-\psi\left(x_{n_{i}}\right)}{\mu_{n_{i}}}\right\rangle-\left\langle\psi(v)-u_{n_{i}}, B x_{n_{i}}\right\rangle \\
&+\frac{\alpha_{n_{i}}}{\mu_{n_{i}}}\left\langle\psi(v)-u_{n_{i}}, \delta \varphi\left(x_{n_{i}}\right)-\psi\left(x_{n_{i}}\right)+\mu_{n_{i}} B x_{n_{i}}\right\rangle \\
& \geq-\left\langle\psi(v)-u_{n_{i}}, \frac{u_{n_{i}}-\psi\left(x_{n_{i}}\right)}{\mu_{n_{i}}}\right\rangle-\left\langle\psi\left(x_{n_{i}}\right)-u_{n_{i}}, B x_{n_{i}}\right\rangle \\
&+\frac{\alpha_{n_{i}}}{\mu_{n_{i}}}\left\langle\psi(v)-u_{n_{i}}, \delta \varphi\left(x_{n_{i}}\right)-\psi\left(x_{n_{i}}\right)+\mu_{n_{i}} B x_{n_{i}}\right\rangle .
\end{aligned}
$$

Since $\left\|\psi\left(x_{n_{i}}\right)-u_{n_{i}}\right\| \rightarrow 0$ and $\psi\left(x_{n_{i}}\right) \rightarrow \psi(z)$, we deduce that $\langle\psi(v)-\psi(z), w\rangle \geq 0$ by taking $i \rightarrow \infty$ in (3.10). Thus, $z \in R^{-1} 0$ by the maximal $\psi$-monotonicity of $R$. Hence, 
$z \in \operatorname{GVI}(B, \psi, C)$. Therefore, $z \in S$. From (3.7), we obtain

$$
\begin{aligned}
& \limsup _{n \rightarrow \infty}\left\langle\delta \varphi\left(x^{*}\right)-\psi\left(x^{*}\right), u_{n}-\psi\left(x^{*}\right)\right\rangle \\
& \quad=\lim _{i \rightarrow \infty}\left\langle\delta \varphi\left(x^{*}\right)-\psi\left(x^{*}\right), \psi\left(x_{n_{i}}\right)-\psi\left(x^{*}\right)\right\rangle \\
& \quad=\left\langle\delta \varphi\left(x^{*}\right)-\psi\left(x^{*}\right), \psi(z)-\psi\left(x^{*}\right)\right\rangle \leq 0 .
\end{aligned}
$$

Note that

$$
\begin{aligned}
\left\|u_{n}-\psi\left(x^{*}\right)\right\| \leq & \left\langle\alpha_{n}\left(\delta \varphi\left(x_{n}\right)-\psi\left(x^{*}\right)\right)+\left(1-\alpha_{n}\right) y_{n}, u_{n}-\psi\left(x^{*}\right)\right\rangle \\
\leq & \alpha_{n} \delta\left\langle\varphi\left(x_{n}\right)-\varphi\left(x^{*}\right), u_{n}-\psi\left(x^{*}\right)\right\rangle+\alpha_{n}\left\langle\delta \varphi\left(x^{*}\right)-\psi\left(x^{*}\right), u_{n}-\psi\left(x^{*}\right)\right\rangle \\
& +\left(1-\alpha_{n}\right)\left\|\psi\left(x_{n}\right)-\mu_{n} B x_{n}-\left(\psi\left(x^{*}\right)-\mu_{n} B x^{*}\right)\right\|\left\|u_{n}-\psi\left(x^{*}\right)\right\| \\
\leq & \alpha_{n} L \delta\left\|x_{n}-x^{*}\right\|\left\|u_{n}-\psi\left(x^{*}\right)\right\|+\alpha_{n}\left\langle\delta \varphi\left(x^{*}\right)-\psi\left(x^{*}\right), u_{n}-\psi\left(x^{*}\right)\right\rangle \\
& +\left(1-\alpha_{n}\right)\left\|\psi\left(x_{n}\right)-\psi\left(x^{*}\right)\right\|\left\|u_{n}-\psi\left(x^{*}\right)\right\| \\
\leq & \alpha_{n}(\delta L / \gamma)\left\|\psi\left(x_{n}\right)-\psi\left(x^{*}\right)\right\|\left\|u_{n}-\psi\left(x^{*}\right)\right\| \\
& +\alpha_{n}\left\langle\delta \varphi\left(x^{*}\right)-\psi\left(x^{*}\right), u_{n}-\psi\left(x^{*}\right)\right\rangle \\
& +\left(1-\alpha_{n}\right)\left\|\psi\left(x_{n}\right)-\psi\left(x^{*}\right)\right\|\left\|u_{n}-\psi\left(x^{*}\right)\right\| \\
= & {\left[1-(1-L \delta / \gamma) \alpha_{n}\right]\left\|\psi\left(x_{n}\right)-\psi\left(x^{*}\right)\right\|\left\|u_{n}-\psi\left(x^{*}\right)\right\| } \\
& +\alpha_{n}\left\langle\delta \varphi\left(x^{*}\right)-\psi\left(x^{*}\right), u_{n}-\psi\left(x^{*}\right)\right\rangle \\
= & \frac{1-(1-L \delta / \gamma) \alpha_{n}\left\|\psi\left(x_{n}\right)-\psi\left(x^{*}\right)\right\|^{2}+\frac{1}{2}\left\|u_{n}-\psi\left(x^{*}\right)\right\|^{2}}{2} \\
& +\alpha_{n}\left\langle\delta \varphi\left(x^{*}\right)-\psi\left(x^{*}\right), u_{n}-\psi\left(x^{*}\right)\right\rangle .
\end{aligned}
$$

It follows that

$$
\begin{aligned}
\left\|u_{n}-\psi\left(x^{*}\right)\right\|^{2} \leq & {\left[1-(1-L \delta / \gamma) \alpha_{n}\right]\left\|\psi\left(x_{n}\right)-\psi\left(x^{*}\right)\right\|^{2} } \\
& +2 \alpha_{n}\left\langle\delta \varphi\left(x^{*}\right)-\psi\left(x^{*}\right), u_{n}-\psi\left(x^{*}\right)\right\rangle .
\end{aligned}
$$

Therefore,

$$
\begin{aligned}
\left\|\psi\left(x_{n+1}\right)-\psi\left(x^{*}\right)\right\|^{2} \leq & \beta_{n}\left\|\psi\left(x_{n}\right)-\psi\left(x^{*}\right)\right\|^{2}+\left(1-\beta_{n}\right)\left\|u_{n}-\psi\left(x^{*}\right)\right\|^{2} \\
\leq & \beta_{n}\left\|\psi\left(x_{n}\right)-\psi\left(x^{*}\right)\right\|^{2} \\
& +\left(1-\beta_{n}\right)\left[1-(1-\delta L / \gamma) \alpha_{n}\right]\left\|\psi\left(x_{n}\right)-\psi\left(x^{*}\right)\right\|^{2} \\
& +2\left(1-\beta_{n}\right) \alpha_{n}\left\langle\delta \varphi\left(x^{*}\right)-\psi\left(x^{*}\right), u_{n}-\psi\left(x^{*}\right)\right\rangle \\
= & {\left[1-(1-\delta L / \gamma)\left(1-\beta_{n}\right) \alpha_{n}\right]\left\|\psi\left(x_{n}\right)-\psi\left(x^{*}\right)\right\|^{2} } \\
& +2\left(1-\beta_{n}\right) \alpha_{n}\left\langle\delta \varphi\left(x^{*}\right)-\psi\left(x^{*}\right), u_{n}-\psi\left(x^{*}\right)\right\rangle \\
= & {\left[1-(1-\delta L / \gamma)\left(1-\beta_{n}\right) \alpha_{n}\right]\left\|\psi\left(x_{n}\right)-\psi\left(x^{*}\right)\right\|^{2} } \\
& +(1-\delta L / \gamma)\left(1-\beta_{n}\right)
\end{aligned}
$$




$$
\begin{aligned}
& \times \alpha_{n}\left(\frac{2}{1-\delta L / \gamma}\left\langle\delta \varphi\left(x^{*}\right)-\psi\left(x^{*}\right), u_{n}-\psi\left(x^{*}\right)\right\rangle\right) \\
= & \left(1-\gamma_{n}\right)\left\|\psi\left(x_{n}\right)-\psi\left(x^{*}\right)\right\|^{2}+\delta_{n} \gamma_{n},
\end{aligned}
$$

where $\gamma_{n}=(1-\delta L / \gamma)\left(1-\beta_{n}\right) \alpha_{n}$ and $\delta_{n}=\frac{2}{1-\delta L / \gamma}\left\langle\delta \varphi\left(x^{*}\right)-\psi\left(x^{*}\right), u_{n}-\psi\left(x^{*}\right)\right\rangle$. It is easily seen that $\sum_{n} \gamma_{n}=\infty$ and $\limsup _{n \rightarrow \infty} \delta_{n} \leq 0$. We can therefore apply Lemma 2.4 to conclude that $\psi\left(x_{n}\right) \rightarrow \psi\left(x^{*}\right)$ and $x_{n} \rightarrow x^{*}$. This completes the proof.

\section{Competing interests}

The authors declare that they have no competing interests.

\section{Authors' contributions}

All authors read and approved the final manuscript.

\section{Author details}

'Department of Mathematics, Tianjin Polytechnic University, Tianjin, 300387, China. ${ }^{2}$ Department of Information Management, Cheng Shiu University, Kaohsiung, 833, Taiwan.

\section{Acknowledgements}

Yonghong Yao was supported in part by NSFC 11071279 and NSFC 71161001-G0105. Rudong Chen was supported in part by NSFC 11071279. Yeong-Cheng Liou was supported in part by NSC 101-2628-E-230-001-MY3 and NSC 101-2622-E-230-005-CC3.

Received: 11 October 2012 Accepted: 14 May 2013 Published: 29 May 2013

\section{References}

1. Censor, Y, Elfving, T: A multiprojection algorithm using Bregman projections in a product space. Numer. Algorithms 8 , 221-239 (1994)

2. Censor, Y, Bortfeld, T, Martin, B, Trofimov, A: A unified approach for inversion problems in intensity modulated radiation therapy. Phys. Med. Biol. 51, 2353-2365 (2006)

3. Censor, Y, Elfving, T, Kopf, N, Bortfeld, T: The multiple-sets split feasibility problem and its applications for inverse problems. Inverse Probl. 21, 2071-2084 (2005)

4. Byrne, C: A unified treatment of some iterative algorithms in signal processing and image reconstruction. Inverse Probl. 20, 103-120 (2004)

5. Yang, Q: The relaxed CQ algorithm for solving the split feasibility problem. Inverse Probl. 20, 1261-1266 (2004)

6. Qu, B, Xiu, N: A note on the CQ algorithm for the split feasibility problem. Inverse Probl. 21, 1655-1665 (2005)

7. Zhao, J, Yang, Q: Several solution methods for the split feasibility problem. Inverse Probl. 21, 1791-1799 (2005)

8. Xu, HK: A variable Krasnosel'skii-Mann algorithm and the multiple-set split feasibility problem. Inverse Probl. 22 2021-2034 (2006)

9. Dang, Y, Gao, Y: The strong convergence of a KM-CQ-like algorithm for a split feasibility problem. Inverse Probl. 27, 015007 (2011)

10. Wang, F, Xu, HK: Approximating curve and strong convergence of the CQ algorithm for the split feasibility problem. J. Inequal. Appl. (2010). doi:10.1155/2010/102085

11. Xu, HK: Iterative methods for the split feasibility problem in infinite-dimensional Hilbert spaces. Inverse Probl. 26, 105018 (2010)

12. Yao, Y, Kim, TH, Chebbi, S, Xu, HK: A modified extragradient method for the split feasibility and fixed point problems J. Nonlinear Convex Anal. 13(3), 383-396 (2012)

13. Yao, Y, Liou, YC, Shahzad, N: A strongly convergent method for the split feasibility problem. Abstr. Appl. Anal. 2012, Article ID 125046 (2012)

14. Stampacchia, G: Formes bilineaires coercivites sur les ensembles convexes. C. R. Math. Acad. Sci. Paris 258, $4413-4416$ (1964)

15. Korpelevich, GM: An extragradient method for finding saddle points and for other problems. Èkon. Mat. Metody 12, 747-756 (1976)

16. Glowinski, R: Numerical Methods for Nonlinear Variational Problems. Springer, New York (1984)

17. Iusem, AN: An iterative algorithm for the variational inequality problem. Comput. Appl. Math. 13, 103-114 (1994)

18. Noor, MA: Some development in general variational inequalities. Appl. Math. Comput. 152, 199-277 (2004)

19. Facchinei, F, Pang, JS: Finite-Dimensional Variational Inequalities and Complementarity Problems. Springer Series in Operations Research, vol. I. Springer, New York (2003)

20. Facchinei, F, Pang, JS: Finite-Dimensional Variational Inequalities and Complementarity Problems. Springer Series in Operations Research, vol. II. Springer, New York (2003)

21. Xu, HK, Kim, TH: Convergence of hybrid steepest-descent methods for variational inequalities. J. Optim. Theory Appl. $119,185-201$ (2003)

22. Yao, JC: Variational inequalities with generalized monotone operators. Math. Oper. Res. 19, $691-705$ (1994)

23. Ceng, LC, Yao, JC: Strong convergence theorem by an extragradient method for fixed point problems and variational inequality problems. Taiwan. J. Math. 10, 1293-1303 (2006) 
24. Ceng, LC, Al-Homidan, S, Ansari, QH, Yao, JC: An iterative scheme for equilibrium problems and fixed point problems of strict pseudo-contraction mappings. J. Comput. Appl. Math. 223, 967-974 (2009)

25. Blum, E, Oettli, W: From optimization and variational inequalities to equilibrium problems. Math. Stud. 63, 123-145 (1994)

26. Combettes, PL, Hirstoaga, A: Equilibrium programming in Hilbert spaces. J. Nonlinear Convex Anal. 6, 117-136 (2005)

27. Yao, Y, Cho, YJ, Liou, YC: Algorithms of common solutions for variational inclusions, mixed equilibrium problems and fixed point problems. Eur. J. Oper. Res. 212, 242-250 (2011)

28. Qin, X, Cho, SY, Kang, SM: Some results on fixed points of asymptotically strict quasi- $\phi$-pseudocontractions in the intermediate sense. Fixed Point Theory Appl. 2012, Article ID 143 (2012)

29. Zegeye, H, Shahzad, N, Alghamdi, MA: Strong convergence theorems for a common point of solution of variational inequality, solutions of equilibrium and fixed point problems. Fixed Point Theory Appl. 2012, Article ID 119 (2012). doi:10.1186/1687-1812-2012-119

30. Qin, X, Agarwal, RP, Cho, SY, Kang, SM: Convergence of algorithms for fixed points of generalized asymptotically quasi- $\psi$-nonexpansive mappings with applications. Fixed Point Theory Appl. 2012, Article ID 58 (2012). doi:10.1186/1687-1812-2012-58

31. Browder, FE: Convergence of approximation to fixed points of nonexpansive nonlinear mappings in Hilbert spaces. Arch. Ration. Mech. Anal. 24, 82-90 (1967)

32. Halpern, B: Fixed points of nonexpansive maps. Bull. Am. Math. Soc. 73, 957-961 (1967)

33. Geobel, K, Kirk, WA: Topics in Metric Fixed Point Theory. Cambridge Studies in Advanced Mathematics, vol. 28 Cambridge University Press, Cambridge (1990)

34. Lions, PL: Approximation de points fixes de contractions. C. R. Hebd. Séances Acad. Sci., Sér. A, Sci. Math. 284 1357-1359 (1977)

35. Opial, Z: Weak convergence of the sequence of successive approximations of nonexpansive mappings. Bull. Am. Math. Soc. 73, 595-597 (1967)

36. Wittmann, R: Approximation of fixed points of non-expansive mappings. Arch. Math. 58, 486-491 (1992)

37. Moudafi, A: Viscosity approximation methods for fixed-points problems. J. Math. Anal. Appl. 241, 46-55 (2000)

38. Xu, HK: Viscosity approximation methods for nonexpansive mappings. J. Math. Anal. Appl. 298, 279-291 (2004)

39. Suzuki, T: Strong convergence theorems for infinite families of nonexpansive mappings in general Banach spaces. Fixed Point Theory Appl. 2005, 103-123 (2005)

40. Xu, HK: Iterative algorithms for nonlinear operators. J. Lond. Math. Soc. 2, 1-17 (2002)

41. Zhang, LJ, Chen, JM, Hou, ZB: Viscosity approximation methods for nonexpansive mappings and generalized variational inequalities. Acta Math. Sin. 53, 691-6988 (2010)

doi:10.1186/1687-1812-2013-140

Cite this article as: Yao et al.: Affine algorithms for the split variational inequality and equilibrium problems. Fixed Point Theory and Applications 2013 2013:140.

\section{Submit your manuscript to a SpringerOpen ${ }^{\odot}$ journal and benefit from:}

$\checkmark$ Convenient online submission

Rigorous peer review

- Immediate publication on acceptance

- Open access: articles freely available online

- High visibility within the field

- Retaining the copyright to your article 